\title{
Automated Visual Inspection and its Application on Automated Inspection
}

\author{
ZI Ke-ming \\ College of Mechanical Engineering, Huaihai Institute of \\ Technology \\ Lianyungang Jiangsu, 222005, China
}

\author{
Wang Hua-bing \\ College of Mechanical Engineering, Huaihai Institute of \\ Technology \\ Lianyungang Jiangsu, 222005, China
}

\begin{abstract}
As Automated Visual Inspection (AVI ) is more advantageous than traditional inspection in high precision, non-contact and automation, it has become one of the hotspots in the research of contemporary computer technology. After the introduction of the definition of AVI, the system of typical AVI is discussed, with a focus on its application on automatic inspection. The development of AVI is proposed at last.
\end{abstract}

Key words-Automated Visual Inspection (AVI ); automatic inspection; image acquisition

\section{INTRODUCTION}

With the increasing research on vision mechanism and the rapid development of computer technology, human beings begin to obtain external image information by using cameras and convert it into digital signals, applying computers in the whole process of visual information processing. AVI can obtain a great deal of external information, and is easily integrated with design and processing information. It has been widely used on such occasions as working condition monitor and on-site monitor in industrial and commercial fields. As a means of inspection, AVI is more and more widely-used, and has gradually developed into an emerging inspection technology--- Automated Visual Inspection (AVI) ${ }^{[1]}$.

\section{DEFINITION OF AVI}

AVI refers to the use of computers and auxiliary equipment to realize the human visual function. Based on the perceived two-dimensional images in the three-dimensional world, the physical structure of three-dimensional objects is studied and extracted for realizing the recognition and understanding of external objects and 3D world. The key theoretical methods for VAI are as follows: they are based on geometry, probability, kinematic calculation and 3D reconstruction computation theory of vision, including projective geometry, probability theory and stochastic processes, motion of rigid body, image processing and artificial intelligence and other theories.

The early research on VAI was mainly based on two dimensions, focusing on the 2D image analysis, the classification was realized by the method of pattern recognition. In the1960s, Roberts was the first one to succeed in interpretation of the three-dimensional world, and took an initiative in interpreting the three-dimensional world with two-dimensional images. In the early 1980s, Marr proposed the computation theory of vision, considering the vision process as information processing process. The theory was rapidly developed into the most important theoretical basis for the computer vision, whose core thought was to restore the three-dimensional shape of objects and their spatial location from two-dimensional images. The theory proposed by Marr has been the most perfect theoretical system in the research of computer vision $^{[2]}$.

\section{AVI SYSTEM}

AVI is a non-contacted method for modern inspection by integrating with such technologies as image processing, precision measurement, pattern recognition, artificial intelligence and on the basis of computer vision.

A normal AVI system mainly includes the light source, an optical system, a CCD camera, the image acquisition device, an image processing module and an actuator and other components, and its specific structure is as shown in Figure 1.

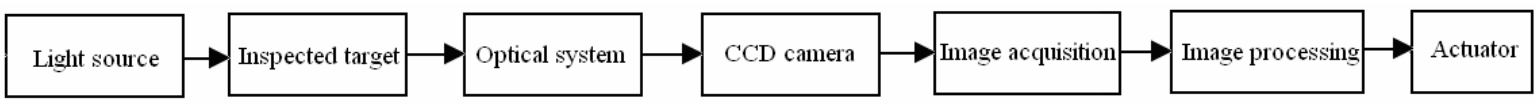

Figure 1 Normal AVI system

AV has been widely used in the manufacturing sector in developed countries since the beginning of 1980s, especially in the repeated inspection of the same products. It has been most widely used in the electronic industry, particularly in the automatic inspection of printed circuit board and integrated circuit chips. Specialized AVI systems are utilized in other industries, such as automobile, packaging, wood and textile industries. As a promising automatic inspection technology, AVI can rapidly inspect the targeted objects with intelligent flexibility and low costs, featuring high precision, on-line and real-time inspection et ${ }^{[3]}$.

\section{IV.APPLICATION OF AVI IN AUTOMATIC INSPECTION}

Automatic inspection is a key element in automatic 
production in the manufacturing industry .The computer vision technology has been widely used in automatic inspection, including geometric measurement and automatic visual inspection of products. The geometric measurement technology is indispensable in product processing in the manufacturing industry. The traditional geometric measurement technology for optical instruments, however, needed complicated reading process and long-time measuring, and there were more subjective errors made by testing people and low automation. The AV is an inspection technology emerging with the development of computer vision technology.

AVI has been applied in inspection of shapes and surface defects of products, such as quality inspection of printing and packing of products, quality inspection of containers, inspection of container filling and sealing, quality inspection of semiconductor IC package and rolled steel, and inspection of key mechanical components. At the customs, the application of $\mathrm{X}$ ray and AVI without opening the cargo inspection greatly improves cargo customs inspection and saves human and material resources, while in pharmaceutical assemble line, AVI can be used for inspection of medicine packaging, so as to make sure the right amount of load or intact pills etc. ${ }^{[4-5]}$.

\section{PROSPECTS OF AVI}

AVI, a promising automatic inspection technology, can rapidly inspect the targeted objects with intelligent flexibility and low costs. It focuses on development in the following three aspects: (1) intelligent inspection, in which product visual model and inspection information are obtained from CAD system, inspection objects, location and path are determined, the intelligent inspection planning is made up ,and industrial robots under control catch spare parts and put them into a suitable location for inspection; (2) on-line and real-time inspection, in which the system can inspect every product, greatly different from the currently-used sampling; (3) high precision inspection, in which the resolution and system errors of CCD camera prevent from the upgrading of precision in computer vision inspection, unable to adapt to inspection of large-sized components ${ }^{[6]}$.

There are also some difficulties in the research and development of AVI at present, which are mainly shown in the following aspects: It is unable to be compatible with the international unified and open standards of practice. The development of calculating theory and new sensors is limited to some extent, and the quantitative model of calculation and the methods for calibration are not perfect.

\section{VI.CONLUSIONS}

AVI has become a new method for automatic inspection, widely used in automatic inspection system in the manufacturing industry. During to its incomparably accurate, efficient and automatic results of inspection, AVI has greatly promoted the development of the manufacturing industry in China. Although there are still some difficulties in the development of AVI, with its future development, it will be further widely applied in automatic inspection in the modern manufacturing industry.

\section{REFERENCES}

[1] Xie Yonghui. Study on Automation Calibration Technology and Accuracy of 3D Laser Scanner System[D]. Huazhong University of Science \& Technology, 2004.

[2] Zhang Yujin. Computer vision [M]. People's Posts and Telecommunications Press, 2011.

[3] Zhang Wen Jing, et al. Computer vision detection technology and its application on inspection of machined parts [J]. Journal of Shanghai Jiao Tong University, 1999, 33 (5): 635 638.

[4] Cai Yejing, et al. Machine vision in packaging testing process applied research [J]. Packaging engineering, 2003, 24 (2): $45 \sim 48$.

[5] ZHANG Xueyi, et al. Development of Automatic Detection System for Medicine Packaging[J]. Packaging engineering, 2009, 30 (6): $47 \sim 50$.

[6] Gao Yingming. Two-dimensional detection system preliminary development on computer vision[D], Dalian University of Technology,2004. 